\title{
The Contribution of Risk Factors to Blood Pressure Heritability Estimates in Young Adults: The East Flanders Prospective Twin Study
}

\author{
Maurice P. A. Zeegers ${ }^{1,2}$, Fruhling Rijsdijk ${ }^{3}$, Pak Sham ${ }^{3,4}$, Robert Fagard ${ }^{5}$, Marij Gielen ${ }^{6}$, Peter W. de Leeuw ${ }^{7}$, \\ and Robert Vlietinck ${ }^{6}$ \\ ' Maastricht University, Department of Epidemiology, Maastricht, the Netherlands \\ ${ }^{2}$ Comprehensive Cancer Institute Limburg, Department of General Practice, Catholic University of Leuven, Leuven, Belgium \\ ${ }^{3}$ Social, Genetic and Developmental Psychiatry Research Centre, Institute of Psychiatry, King's College London, London, United Kingdom \\ ${ }^{4}$ Department of Psychiatry, University of Hong Kong, Queen Mary Hospital, Hong Kong, China \\ ${ }^{5}$ Hypertension and Cardiovascular Rehabilitation Unit, Faculty of Medicine, Catholic University of Leuven, Leuven, Belgium \\ ${ }^{6}$ Department of Population Genetics, Genomics and Bioinformatics, Maastricht University, Maastricht, the Netherlands \\ ${ }^{7}$ Department of Medicine, University Hospital Maastricht, the Netherlands
}

\begin{abstract}
Tress he heritability of blood pressure estimated in previous studies may be confounded by the influence of potential blood pressure risk factors. We applied the classical twin design to estimate the contribution of these covariates to blood pressure heritability. The study consisted of 173 dizygotic and 251 monozygotic twin pairs aged 18-34 years, randomly selected from the East Flanders Prospective Twin Survey. In a standardized examination, blood pressure and anthropometry was measured, a questionnaire was completed, and a fasting blood sample was taken. In univariate and bivariate modeling, diastolic and systolic heritability were estimated both unadjusted and adjusted for potential risk factors. Also, covariate interaction was modeled. Bivariate analysis gave heritability estimates of $0.63(95 \% \mathrm{Cl} 0.55-0.59), 0.74(95 \% \mathrm{Cl}$ : 0.68-0.79), and $0.78(95 \% \mathrm{Cl}: 0.70-0.84)$ for diastolic, systolic, and cross-trait heritability, respectively. The remaining variances could be attributed to unique environmental influences. These heritability estimates did not change substantially in univariate analyses or after adjustment for risk factors. A sex-limitation model showed that the heritability estimates for women were significantly higher than for men, but the same genetic factors were operating across sexes. Sex and cigarette smoking appeared to be statistically significant interaction terms. The heritability of blood pressure is relatively high in young adults. Potential risk factors of blood pressure do not appear to confound the heritability estimates. However, gene by sex by smoking interaction is indicated.
\end{abstract}

Since an elevated blood pressure is a major risk factor for cardiovascular complications such as stroke, myocardial infarction, and peripheral vascular disease it is essential to investigate the origin of blood pressure and to disentangle environmental from genetic influence (Kaplan, 1998). The genetic origin of blood pressure has been examined in several twin studies in the last two decades (Bielen et al., 1991; Carmelli et al., 1991; Carroll et al., 1985; Degaute et al., 1994; Fagard et al., 1995; Feinleib et al., 1977; Hong et al., 1994; Hunt et al., 1989; IJzerman et al., 2000; Manatunga et al., 1992; Schieken et al., 1989; Schieken et al., 1992; Slattery et al., 1988; Smith et al., 1987; Snieder et al., 2000; Tambs et al., 1993; Vinck et al., 1999; Vinck et al., 2001; Williams et al., 1992; Williams et al., 1993). In these studies, the variation in blood pressure that can be attributed to genetic differences has been estimated at 30 to $60 \%$. However, little attention has been paid to measurable potential risk factors for blood pressure. These risk factors may be more important than genetic factors alone in terms of impact on public health because they are more easily modified. The study of twins provides a powerful design to distinguish between environmental factors and genetic influences. Monozygotic (MZ) twins are genetically identical whereas dizygotic (DZ) twins share, on average, half their genes. In the twin design, greater blood pressure similarity in MZ compared to DZ twins is taken as evidence for a genetic basis. However, the estimated genetic influence can be confounded by risk factors for blood pressure when the distribution of these covariates is not equal for $\mathrm{MZ}$

Received 16 June, 2003; accepted 30 March, 2004.

Address for correspondence: Maurice Zeegers, Maastricht University, Department of Epidemiology, PO Box 616, 6200 MD Maastricht, The Netherlands.Email: mpa.zeegers@epid.unimaas.nl 
and DZ twins. Also, the effect of risk factors may be expressed as gene by covariate interaction, indicated by different heritability estimates across subgroups of individuals with certain risk factor profiles. Although of potential importance, most previous heritability studies did not investigate the influence of specific risk factors on blood pressure heritability estimates.

In previous analyses, we have suggested that Body Mass Index (BMI; Vinck et al., 1999) and age (Vinck et al., 2001) may influence the estimation of blood pressure heritability. However, the analyses were based on a small number of twin pairs, no extensive modeling of various potential risk factors was performed, and no gene by covariate interaction was investigated. Therefore, in the present study, we have examined the influence of age, BMI, cholesterol level, birthweight, physical exercise, sex, and cigarette smoking on the heritability of diastolic and systolic blood pressure in more detail on a larger sample of twin pairs.

\section{Methods \\ Participants}

The study sample consisted of 424 twin pairs between 18 and 34 years of age, who were randomly selected from the East Flanders Prospective Twin Survey (Loos et al., 1998). This population-based survey has prospectively registered all twins born in the Belgian Province of East Flanders since 1964. Zygosity was determined through sequential analysis based on sex, fetal membranes, umbilical cord blood groups, placental alkaline phosphatase, and DNA fingerprints. Between July 1964 and May 1982, the Twin Survey had registered 2141 twin pairs who met the World Health Organization criteria for live born infants (birthweight $\geq 500 \mathrm{~g}$ or gestational age $\geq 22$ weeks, if birthweight unknown). Pairs of whom one or both members were stillborn, died in neonatal or later life, or suffered from major congenital malformation were excluded. We randomly contacted 803 pairs using an envelope system (randomly drawing identification numbers out of an envelope). To assure equally distributed groups, we stratified for birth year and zygosity. Since the twin survey was originally engaged in the determination of chorionicity, the number of $\mathrm{MZ}$ twin pairs was over sampled. Four hundred and twenty-four representative twin pairs $(52.8 \%)$ volunteered to participate in the current study of whom 173 were DZ (56 female, 60 male, and 57 opposite sex pairs) and 251 were MZ (132 female and 119 male pairs). The twins gave informed consent and the project was approved by the Local Committee of Medical Ethics. The twins have been labeled twin 1 and twin 2 for the first and second born twin member, respectively. However, for analytical reasons, in DZ opposite sex pairs, twin 1 always represents the female twin member.

\section{Measures}

Perinatal data including birth date, sex, and birthweight were prospectively registered at birth. The newborns were weighed to the nearest $100 \mathrm{~g}$ by the pediatrician or midwife. Between February 1997 and April 2000, all twins visited our laboratory for a 2hour examination in the morning. The twins separately filled out a questionnaire on risk factors including current smoking frequency and physical activity. After 5 minutes of supine rest, blood pressure $(\mathrm{mmHg})$ was measured in supine and sitting position on the right arm in triplicate by sphygmomanometry and auscultation (Korotkoff phases I and V) by one of four trained investigators. The reported resting blood pressure is the average of these three measurements. In addition, blood pressure was measured after 1 and 2 minutes of hand gripping. The reported blood pressure is the average of these two measurements. Standing height $(\mathrm{cm})$ was measured with a Harpenden fixed stadiometer to the nearest $0.1 \mathrm{~cm}$ and body mass $(\mathrm{kg})$ was measured on a balance scale (SECA) to the nearest $0.1 \mathrm{~kg}$. BMI was calculated as body mass divided by squared height. A fasting blood sample was taken to determine the concentrations of cholesterol $(\mathrm{mg} / \mathrm{dL})$. The reported cholesterol measure is the ratio of total cholesterol and HDL cholesterol.

\section{Statistical Analysis}

Means, standard deviations, and Pearson correlations with diastolic and systolic blood pressure were calculated by zygosity strata for each risk factor to estimate their relationship with blood pressure variability and to identify potential confounders. Also, zygosity-stratified mean blood pressure and withintwin pair intraclass correlations were calculated to investigate genetic influence. To determine whether blood pressure measured in supine and sitting position and after hand gripping represent a similar construct (i.e., diastolic or systolic blood pressure) principal components factor analyses followed by varimax rotation were performed.

Variance components were estimated by using the structural equation modeling program $\mathrm{Mx}$ (Neale et al., 2002). This technique is based on the comparison of the variance-covariance matrices in $\mathrm{MZ}$ and $\mathrm{DZ}$ twin pairs and allows partitioning of the observed phenotypic variance into latent additive genetic (A), shared $(\mathrm{C})$, or unique $(\mathrm{E})$ environmental components. The latter also contains the measurement error. Dividing each of these components of variance by the total variance yields the different standardized components of variance; for example, the narrow sense heritability $\left(\mathrm{h}^{2}\right)$, which can be defined as the ratio of additive genetic variance to total phenotypic variance. We did not investigate a dominant genetic variance component (D) since AD, DE, or ADE models would have yielded to underpowered results. In univariate analyses the influence of the above mentioned variance components were estimated separately for diastolic and systolic blood pressure.

The genetic structure of diastolic and systolic blood pressure (and their correlation) was also analyzed 
in a bivariate genetic model. In the bivariate model the $\mathrm{A}, \mathrm{C}$, and $\mathrm{E}$ matrices are specified in a triangular (Cholesky) decomposition. This decomposition implies as many (genetic and environmental) latent factors as dependent variables. In, for example, the genetic matrix, factor A1 influences both diastolic and systolic blood pressure, whereas factor A2 only influences the systolic blood pressure (Figure 1). The variance components for each phenotype were estimated, as well as the genetic, common environmental, and unique environmental correlations between phenotypes by standardizing the A, C, and $\mathrm{E}$ matrices, respectively. In addition, we partitioned the phenotypic correlation between phenotypes in parts determined by common genetic, common shared, and unique environmental effects. The genetic contribution to the phenotypic correlation (cross-trait heritability) was calculated by $h_{1} \times r_{g} \times h_{2}$, where $r_{g}$ is the genetic correlation and $h_{1}$ and $h_{2}$ are standardized additive genetic path estimates for diastolic and systolic blood pressure, respectively.

The statistical significance of the variance component models was assessed by testing the deterioration in fit of sub-models in which either A or C were dropped from the full (ACE) model. Sub-models were compared with the full model by hierarchical $\chi^{2}$ tests. The difference in $\chi^{2}$ values between sub-model and full model is itself approximately distributed as $\chi^{2}$, with degrees of freedom $(d f)$ equal to the difference in $d f$ of the sub-model and the full model. Model selection was also guided by Akaike's information criterion (AIC $=\chi^{2}-2 d f$ ) when compared to the saturated model (Aikaike, 1987). The model with the lowest AIC reflects the best balance between goodness of fit and parsimony.

All variance components were estimated both unadjusted and adjusted for the covariates age, BMI, cholesterol ratio, and sex. In adjusted analyses, the influences of these risk factors are simultaniously accounted for in the expected means model of the saturated and genetic models as definition variables (Neale et al., 2002). Figure 1 shows the path diagram of a mean-adjusted triangular decomposition bivariate model for the two blood pressure phenotypes included in the analysis. This method is equivalent to the incorporation of risk factors in the covariance model (Zeegers et al., 2004). The unadjusted and adjusted variance components estimates with corresponding $95 \%$ confidence intervals for the most parsimonious model are presented.

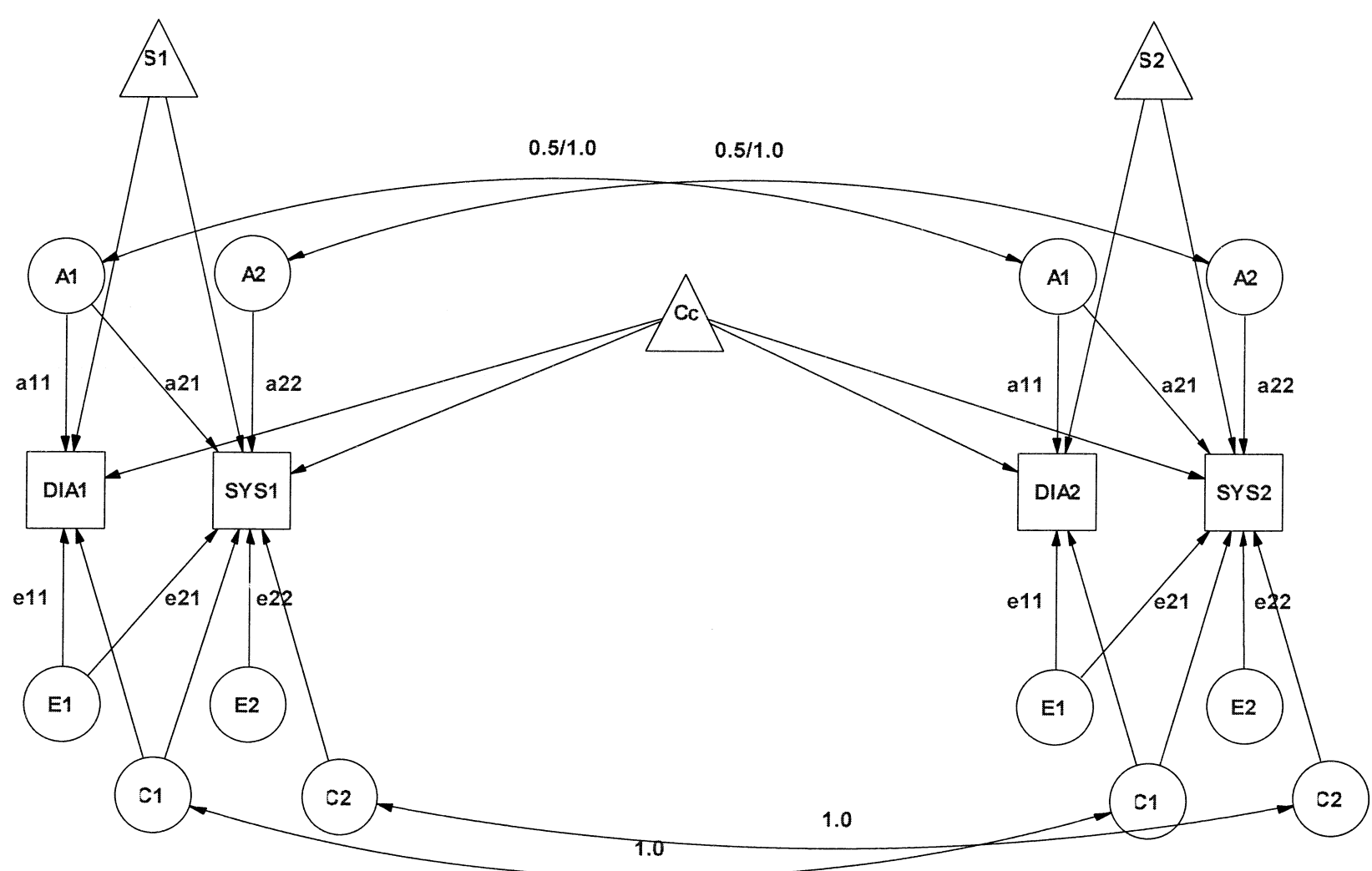

Figure 1

Path diagram of Cholesky bivariate twin analysis on diastolic and systolic blood pressure with mean-adjustment.

Note: Squares represent observed variables, including diastolic (DIA1/2) and systolic (SYS1/2) blood pressure. Circles represent latent variables, including additive genetic (A1/2) common environment (C1/2), and unique environment (E1/2) components. Triangles represent definition variables which were incorporated in the means model, including twin specific covariates ( $\mathrm{Sc} 1 / 2$ ) and common covariates (Cc). Variables were numbered 1 or 2 for twin 1 or 2, respectively. 


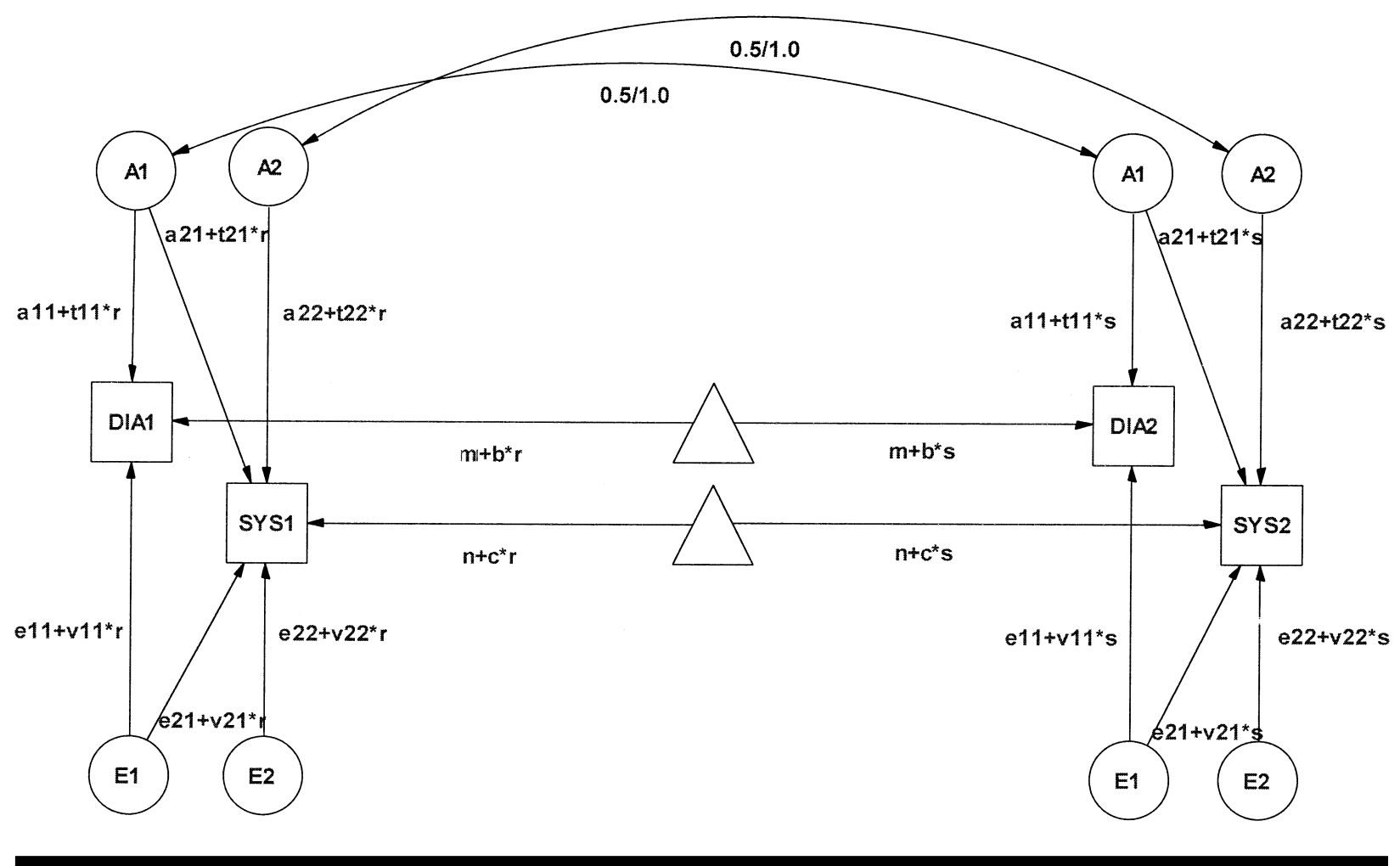

\section{Figure 2}

Path diagram of Cholesky bivariate twin analysis on diastolic and systolic blood pressure with covariate interaction.

Note: Squares represent observed variables, including diastolic (DIA1/2) and systolic (SYS1/2) blood pressure. Circles represent latent variables, including additive genetic (A1/2) and unique environment (E1/2) components. The paths are modeled by linear regression equations with an additive genetic intercept (A) and slope ( $T$ ), a unique environment intercept (E) and slope (V), and a vector of twin specific covariates for twin 1 ( $r$ ) and twin 2 (s). Variables were numbered 1 or 2 for twin 1 or 2 , respectively.

In addition to the investigation of potential main covariate confounding effects, gene by covariate interaction (i.e., change in heritability as a function of the covariate level) is evaluated for age, BMI, cholesterol ratio, birthweight, physical activity, sex, and the natural logarithm of cigarette smoking. Sex differences in heritability were investigated in a standard bivariate unadjusted triangular decomposition sexlimitation model with additive genetic and unique environmental variance components (Neale \& Cardon, 1992). Quantitative sex differences (i.e., difference in heritability estimates) were measured by comparing models in which variance components were separately estimated for men and women to models in which they were equated across sexes. Qualitative sex differences (i.e., whether the same or different genes are related to blood pressure in men and women) were estimated by testing whether the genetic correlation in opposite sex pairs (57 pairs) was significantly different from .5 .

Gene by covariate interaction (i.e., if heritable variance varies as a function of the value on a continuous covariate) was tested using the entire distribution instead of classes of observations. In this approach, the genetic and environmental path coefficients have a constant part and a variable part that depends linearly on the twin specific risk factors, which are modeled as definition variables in $\mathrm{Mx}$ (Figure 2; Purcell, 2002). The significance of the gene by covariate interaction was calculated by testing the fall in $\chi^{2}$ after dropping the covariate effect from the full model. Those covariates that yielded significant interaction terms were subsequently modeled together to investigate their combined influence.

Although, data on chorionicity was available, we did not take chorionicity into account because the heritability estimates of blood pressure did not differ significantly according to the chorionicity of the monozygotic twins (Fagard et al., 2003).

\section{Results}

Characteristics of the Study Population

The means, standard deviations and Pearson correlations of the risk factors with diastolic and systolic blood pressure are shown in Table 1 for each risk factor, stratified by zygosity status group. The mean age of DZ and MZ twins was 26.47 and 25.30 years, respectively. birthweight, physical activity and cigarette smoking did not differ substantially across zygosity status. Age, BMI, cholesterol ratio and sex appeared to be correlated with diastolic and systolic blood pressure and were normally distributed. Therefore the latter untransformed covariates were incorporated in subsequent adjusted analyses. 
Table 1

Means, Standard Deviations and Pearson Correlations with Diastolic and Systolic Blood Pressure for Each Covariate, Stratified by Zygosity Status; East Flanders Prospective Twin Study

\begin{tabular}{|c|c|c|c|c|c|c|c|c|}
\hline \multirow[t]{2}{*}{ Continuous covariates } & \multicolumn{4}{|c|}{ Dizygotic Twin Pairs ( $n=173$ ) } & \multicolumn{4}{|c|}{ Monozygotic Twin Pairs $(n=251)$} \\
\hline & $M^{\mathrm{a}}$ & $S^{b}$ & $R_{\mathrm{d}}^{\mathrm{c}}$ & $R_{\mathrm{s}}^{\mathrm{d}}$ & $M^{\mathrm{a}}$ & $S^{\mathrm{b}}$ & $R_{\mathrm{d}}^{\mathrm{c}}$ & $R_{\mathrm{s}}^{\mathrm{d}}$ \\
\hline Age (years) & 26.47 & 4.78 & .18 & -.01 & 25.30 & 4.62 & .09 & .00 \\
\hline Body Mass Index & 22.24 & 2.92 & .31 & .30 & 22.23 & 3.26 & .29 & .32 \\
\hline Cholesterol ratio $^{\mathrm{e}}$ & 3.42 & 1.06 & .32 & .20 & 2.49 & 0.45 & .31 & .28 \\
\hline Birthweight (kg) & 2.61 & 0.43 & -.02 & -.12 & 4.88 & 1.77 & -.03 & .00 \\
\hline Physical activity score (0-10) & 5.05 & 1.89 & -.05 & .00 & 10.45 & 6.89 & .02 & .09 \\
\hline Discrete covariates & $N^{\mathrm{f}}$ & $\%$ & $R_{\mathrm{d}}^{\mathrm{c}}$ & $R_{\mathrm{s}}^{\mathrm{d}}$ & $N^{\mathrm{f}}$ & $\%$ & $R_{\mathrm{d}}^{\mathrm{c}}$ & $R_{\mathrm{s}}^{\mathrm{d}}$ \\
\hline \multicolumn{9}{|l|}{ Sex } \\
\hline Female & 56 & 32.37 & & & 132 & 52.59 & & \\
\hline Male & 60 & 34.68 & & & 119 & 47.41 & & \\
\hline Opposite sex & 57 & 32.95 & .27 & .26 & & & .38 & .47 \\
\hline \multicolumn{9}{|l|}{ Current cigarette smoking } \\
\hline No & 24 & 17.27 & & & 52 & 21.58 & & \\
\hline Yes & 65 & 46.76 & & & 152 & 63.07 & & \\
\hline Opposite smoking status ${ }^{\mathrm{g}}$ & 50 & 35.97 & 0.00 & 0.05 & 37 & 15.35 & .07 & .10 \\
\hline  & $\begin{array}{l}\text { ood pres } \\
\text { od press } \\
\end{array}$ & & & & & & & \\
\hline
\end{tabular}

Both across twins and between zygosity strata, the mean diastolic and systolic blood pressure values in supine and sitting position and after hand gripping were each similar (Table 2). For all phenotypes, the intraclass correlations in $\mathrm{MZ}$ twin pairs were approximately twice as high as those in DZ twin pairs, indicating possible additive genetic influences (Table 2).

Principal components analysis after varimax rotation revealed that all blood pressure measurements could be summarized by the two factors alone, which explained almost $90 \%$ of the total variation in blood pressure (Table 3). This factor indicates a dummyvariable for diastolic or systolic blood pressure. Although clinically different, the method of blood pressure measurement - in supine or sitting position or after hand gripping - did not yield to numerically distinguishable factors. Therefore, the mean diastolic blood pressure and the mean systolic blood pressure across types of measurements were used in variance components analysis. These two phenotypes serve as indicators for the different postures in blood pressure measurement. The cross-twin correlations for mean diastolic blood pressure were .68 and .21 for monozygotic and dizygotic twins. For systolic blood pressure these correlations were .67 and .35 , respectively.

\section{Univariate Analysis}

Unadjusted univariate analysis showed that for diastolic and systolic blood pressure both ACE and AE models explained the phenotypic variance equally well. The corresponding AIC were 3.73 and 1.73 for diastolic blood pressure and 3.35 and 1.49 for systolic blood pressure, favoring the AE model. The results from adjusted univariate analysis did not differ substantially. The unadjusted and adjusted heritability estimates of diastolic blood pressure were 0.64 (95\% CI: $0.57-0.70)$ and 0.60 (95\% CI: 0.51$0.67)$, respectively. The unadjusted and adjusted heritability estimates of systolic blood pressure were 0.74 (95\% CI: $0.68-0.79)$ and 0.70 (95\% CI: 0.63-0.75), respectively.

\section{Bivariate Analysis}

Results of bivariate triangular model fitting are shown in Table 4 . Hierarchical $\chi^{2}$ tests showed that the fit of the AE model was not significantly different from that of the ACE model $\left(p_{\text {unadjusted }}=.93\right.$ and $p_{\text {adjusted }}$ $=1.00)$. The AIC confirmed that the AE model was the most parsimonious solution. Diastolic and systolic variance components of the best-fitting bivariate model were virtually equal to the results of the univariate modeling: the unadjusted and adjusted diastolic heritability estimates were $0.63(95 \% \mathrm{CI}$ 0.55-0.59) and 0.59 (95\%CI: 0.51-0.67; Table 4). The systolic heritability estimates were 0.74 (95\% CI: $0.68-0.79$ ) without adjustment for risk factors and 0.70 (95\% CI: 0.63-0.75) after adjustment (Table 4). The unadjusted and adjusted cross-trait heritability estimates were estimated to be 0.78 (95\% CI: $0.70-0.84)$ and 0.75 (95\%CI: 0.65-0.83).

\section{Gene by Covariate Interaction}

Since previous analyses showed that the results from unadjusted and adjusted analyses and from univariate and multivariate were virtually equal, only unadjusted triangular multivariate $\mathrm{AE}$ models were 
used to investigate potential interaction caused by environmental risk factors. Sex limitation modeling showed that a model with separate parameter estimates for men and women and a fixed genetic correlation (i.e., .5) in opposite sex pairs was the most parsimonious solution. This finding was confirmed in interaction modeling $(p<.005)$. Also, cigarette smoking $(p=.03)$ showed to be a statistically significant interaction term. Therefore, the effect of sex and cigarette smoking on heritability estimates for diastolic and systolic blood pressure were estimated simultaneously by using a sex-limitation model allowing for continuous interaction modeling for cigarette smoking. The results show that for both men and women the diastolic blood pressure heritability decreases with increasing smoking frequency. However, for systolic blood pressure the results for men and women differ. Although, for men, the systolic blood pressure heritability decreases with increasing smoking frequency, for women, the systolic blood pressure heritability increases with increasing smoking frequency (Figure 3).

\section{$\overline{\text { Discussion }}$}

Few other twin studies have applied similar statistical methods to quantify the contribution of risk factors to blood pressure heritability estimates. In univariate and bivariate structural equation modeling diastolic and systolic heritability were estimated both unadjusted and adjusted for risk factors. Also, gene by covariate interaction was modeled and subgroup-specific heritability estimates were calculated.

The present study confirms that, although familial clustering of blood pressure can be explained by common environmental factors, it is mainly determined by genetic risk factors. Both univariate and bivariate analysis showed consistently that the AE model was the most parsimonious model to explain diastolic and systolic variability, regardless which method was used to measure blood pressure. The heritability estimates were $0.63,0.74$, and 0.78 for diastolic, systolic, and cross-trait heritability, respectively. These relatively high estimates may be due to the fact that a rather homogeneous population of young twins was studied. In this and other studies it has been shown that the influence of heritability decreases with advancing age (Hong et al., 1994; Vinck et al., 2001), although not always statistically significantly (Vinck et al., 2001). The high cross-trait heritability shows that the same genes seem to influence both diastolic and systolic blood pressure. The same genes also seem to act in both men and women since the genetic correlation in opposite sex pairs could be fixed to .5 in sex-limited analyses. However, gene by sex interaction may exist: in our study, the heritability is higher for women than for men. Also, gene by sex by cigarette smoking interaction may exist as is shown in Figure 3. It is, nevertheless, possible that the environmental variance and not the genetic



\section{Figure 3}

Interaction of smoking frequency with diastolic and systolic heritability estimates for females.

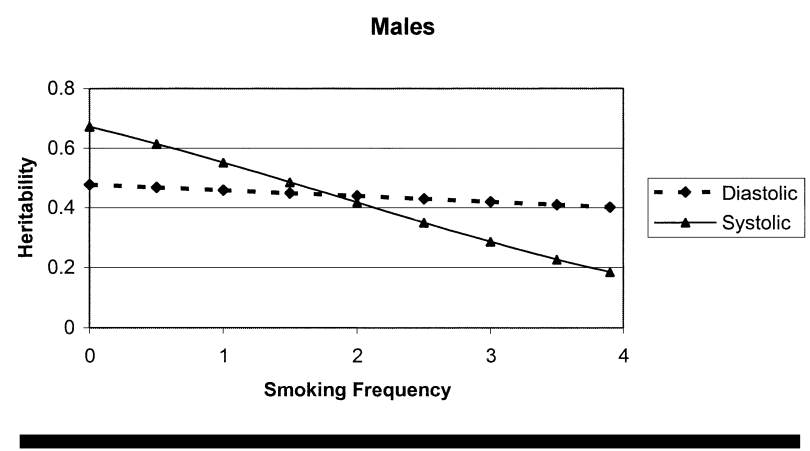

\section{Figure 4}

Interaction of smoking frequency with diastolic and systolic heritability estimates for males.

variance differs between males and females, which can also lead to differences in heritability estimates.

With the exception of monogenetic abnormalities such as Liddle's disease, no evidence has been presented so far for a specific cluster of major hypertension genes. Since blood pressure is the end result of many physiological processes, it is unlikely that a uniform genetic background will ever be found. Instead, abnormalities in candidate genes are far more useful to pursue. In this respect, the most important candidates are those related to the renin-angiotensin system and volume control. Indeed, many studies have shown the impact of polymorphisms in the genes encoding angiotensinogen or the angiotensin II type 1 receptor. Other potentially interesting genes are those encoding alpha- or beta-adducin or G protein (3subunit protein). Blood pressure may be increased especially when an individual has an accumulation of "unfavorable" candidate genes.

All blood pressures were measured by the same investigator and under well-standardized conditions: in the morning, in the same quiet room and after the participants had rested for 5 minutes in the supine position, which may have minimized environmental influences. Whereas similar unique environmental covariances are assumed for the monozygotic and dizygotic twins, environmental covariance may in 
fact be greater in the monozygotic twins (Slattery et al., 1988).

Higher correlation for unique environmental risk factors in monozygotic twins may bias upward the heritability estimates. However, in this investigation BMI, cholesterol ratio, birthweight, physical activity, sex, and cigarette smoking were equally concordant in $M Z$ and DZ twin pairs. Further, we found that the heritability estimates for diastolic and systolic blood pressure were not substantially altered by adjustment for these risk factors. These results are consistent with four previous studies (Fagard et al., 1995; Slattery et al., 1988; Snieder et al., 2000; Vinck et al., 1999), which made an attempt to correct for at least one specific risk factor as well.

The twins were on average 26 years, ranging between 18 and 34 years. The lower limit of 18 years was chosen as a previous meta-analyses has shown that findings on blood pressure heritability during adolescence were inconsistent (Law \& Shiell, 1996), which may be related to the unusual growth dynamics during these phases of growth. The upper limit of 34 years was imposed by the EFPTS, the twin register from which our participants were selected. The EFPTS was founded in 1964. The reported results are likely to be representative of the general study population because basic characteristics of the twins in this investigation were similar to the population-based sample of twins in the EFPTS. Also, a recent study showed that estimates of genetic and environmental contributions to blood pressure deriving from twin studies may be generalized to singletons (de Geus et al., 2001). However, since we were limited to young adults, the results of our study cannot be generalized to older adults.

In this and other twin studies survival of the fittest selection may exist since perinatal mortality and morbidity in twins is significantly higher than in singletons (Kiely, 1990). This is not only due to premature delivery and low birthweight, but being a twin member increases the risk of other adverse outcomes as well (Buekens \& Wilcox, 1993). As for many other twin studies, we relied on volunteer twin databases as healthy twin pairs are most likely to register on these databases. Also, twins who have severe morbidity and who were unable to perform the requested tasks were not included in this study. As a consequence, our study population may represent the healthy survivors and, therefore, they may have a lower risk of developing high blood pressure. Although this population selection may have decreased the statistical power of this study, we expect it to be non-differential within and across twin pairs, indicating that the parameters estimated in this study are probably not biased.

In summary, the present study confirms that blood pressure is significantly genetically determined. The clustering of both diastolic and systolic blood pressure in families appears not to be influenced by common environmental risk factors but mainly by additive genetic factors. The same genes seem to influence both diastolic and systolic blood pressure for both men and women. Potential risk factors appear not to confound the heritability estimates substantially. However, gene by sex by smoking interaction is indicated.

\section{$\overline{\text { References }}$}

Aikaike, H. (1987). Factor analysis and AIC. Psychometrika, 52, 317-332.

Bielen, E. C., Fagard, R. H., \& Amery, A. K. (1991). Inheritance of blood pressure and haemodynamic phenotypes measured at rest and during supine dynamic exercise. Journal of Hypertension, 9, 655-663.

Buekens, P., \& Wilcox, A. (1993). Why do small twins have a lower mortality rate than small singletons? American Journal of Obstetrics and Gynecology, 168(3 Pt 1), 937-941.

Carmelli, D., Ward, M. M., Reed, T., Grim, C. E., Harshfield, G. A., \& Fabsitz, R. R. (1991). Genetic effects on cardiovascular responses to cold and mental activity in late adulthood. American Journal of Hypertension, 4(3 Pt 1), 239-244.

Carroll, D., Hewitt, J. K., Last, K. A., Turner, J. R., \& Sims, J. (1985). A twin study of cardiac reactivity and its relationship to parental blood pressure. Physiology and Behavior, 34, 103-106.

Degaute, J. P., Van Cauter, E., van de Borne, P., \& Linkowski, P. (1994). Twenty-four-hour blood pressure and heart rate profiles in humans. A twin study. Hypertension, 23, 244-253.

de Geus, E. J., Posthuma, D., Ijzerman, R. G., \& Boomsma, D. I. (2001). Comparing blood pressure of twins and their singleton siblings: Being a twin does not affect adult blood pressure. Twin Research, 4, 385-391.

Fagard, R., Brguljan, J., Staessen, J., Thijs, L., Derom, C., Thomis, M., et al. (1995). Heritability of conventional and ambulatory blood pressures. A study in twins. Hypertension, 26(6 Pt 1), 919-924.

Fagard, R. H., Loos, J. F., Beunen, G., Derom, C., \& Vlietinck, R. (2003). Influence of chorionicity on the heritability estimates of blood pressure: A study in twins. Journal of Hypertension, 21, 1313-1318.

Feinleib, M., Garrison, R. J., Fabsitz, R., Christian, J. C., Hrubec, Z., Borhani, N. O., et al. (1977). The NHLBI twin study of cardiovascular disease risk factors: Methodology and summary of results. American Journal of Epidemiology, 106, 284-285.

Hong, Y., de Faire, U., Heller, D. A., McClearn, G. E., \& Pedersen, N. (1994). Genetic and environmental influences on blood pressure in elderly twins. Hypertension, 24, 663-670.

Hunt, S. C., Hasstedt, S. J., Kuida, H., Stults, B. M., Hopkins, P. N., \& Williams, R. R. (1989). Genetic heritability and common environmental components of resting and stressed blood pressures, lipids, and body 
mass index in Utah pedigrees and twins. American Journal of Epidemiology, 129, 625-638.

IJzerman, R., Stehouwer, C. D., \& Boomsma, D. I. (2000). Evidence for genetic factors explaining the birthweight-blood pressure relation. Analysis in twins. Hypertension, 36, 1008-1012.

Kaplan, N. M. (1998). Clinical hypertension (7th ed.). Baltimore: Williams \& Wilkins.

Kiely, J. L. (1990). The epidemiology of perinatal mortality in multiple births. Bulletin of the New York Academy of Medicine, 66, 618-637.

Law, C. M., \& Shiell, A. W. (1996). Is blood pressure inversely related to birthweight? The strength of evidence from a systematic review of the literature. Journal of Hypertension, 14, 935-941.

Loos, R., Derom, C., Vlietinck, R., \& Derom, R. (1998). The East Flanders Prospective Twin Survey (Belgium): A population-based register. Twin Research, 1, 167-175.

Manatunga, A. K., Reister, T. K., Miller, J. Z., \& Pratt, J. H. (1992). Genetic influences on the urinary excretion of aldosterone in children. Hypertension, 19, 192-197.

Neale, M. C., Boker, S. M., Xie, G., \& Maes, H. H. (2002). Mx: Statistical Modeling (Vol. 6). Richmond: Department of Psychiatry.

Neale, M. C., \& Cardon, L. R. (1992). Methodology for genetic studies of twins and families. Dordrecht, the Netherlands: Kluwer Academic Publishers.

Purcell, S. (2002). Variance components models for gene-environment interaction in twin analysis. Twin Research, 5, 554-571.

Schieken, R. M., Eaves, L. J., Hewitt, J. K., Mosteller, M., Bodurtha, J. N., Moskowitz, W. B., et al. (1989). Univariate genetic analysis of blood pressure in children (the Medical College of Virginia Twin Study). The American Journal of Cardiology, 64, 1333-1337.

Schieken, R. M., Mosteller, M., Goble, M. M., Moskowitz, W. B., Hewitt, J. K., Eaves, L. J., et al. (1992). Multivariate genetic analysis of blood pressure and body size. The Medical College of Virginia Twin Study. Circulation, 86, 1780-1788.

Slattery, M. L., Bishop, D. T., French, T. K., Hunt, S. C., Meikle, A. W., \& Williams, R. R. (1988). Lifestyle and blood pressure levels in male twins in Utah. Genetic Epidemiology, 5, 277-287.

Smith, T. W., Turner, C. W., Ford, M. H., Hunt, S. C., Barlow, G. K., Stults, B. M., et al. (1987). Blood pressure reactivity in adult male twins. Health Psychology, 6, 209-220.

Snieder, H., Hayward, C. S., Perks, U., Kelly, R. P., Kelly, P. J., \& Spector, T. D. (2000). Heritability of central systolic pressure augmentation: A twin study. Hypertension, 35, 574-579.

Tambs, K., Eaves, L. J., Moum, T., Holmen, J., Neale, M. C., Naess, S., et al. (1993). Age-specific genetic effects for blood pressure. Hypertension, 22, 789-795.

Vinck, W. J., Fagard, R. H., Loos, R., \& Vlietinck, R. (2001). The impact of genetic and environmental influences on blood pressure variance across agegroups. Journal of Hypertension, 19, 1007-1013.

Vinck, W. J., Vlietinck, R., \& Fagard, R. H. (1999). The contribution of genes, environment and of body mass to blood pressure variance in young adult males. Journal of Human Hypertension, 13, 191-197.

Williams, P. D., Puddey, I. B., Martin, N. G., \& Beilin, L. J. (1992). Platelet cytosolic free calcium concentration, total plasma calcium concentration and blood pressure in human twins: A genetic analysis. Clinical Science (London), 82, 493-504.

Williams, P. D., Puddey, I. B., Martin, N. G., \& Beilin, L. J. (1993). Genetic and environmental covariance of serum cholesterol and blood pressure in female twins. Atherosclerosis, 100, 19-31.

Zeegers, M., Rijsdijk, F., \& Sham, P. (2004). Adjusting for covariates in variance components QTL linkage analysis. Behavior Genetics, 34, 127-133. 